\title{
The Assessment of a "Crossover" Translation Technique for Innovative Development of Central Asia Mining Regions
}

\author{
Roman Islamov ${ }^{1, *}$, and Oksana Greenwald ${ }^{1}$ \\ ${ }^{1}$ Kemerovo State University, Institute of Philology, Foreign Languages and Mediacommunications, \\ Department of Foreign Languages, 650000, 6 Krasnaya st., Kemerovo, Russia
}

\begin{abstract}
Innovative development of mining regions is undoubtedly determined by efficient specialists in mining engineering, ecology and natural resources management. Due to close integration and economic relations of Kemerovo Region, a leading mining region in Russia, with the adjacent states of Central Asia many young people from Tajikistan, Kyrgyzstan and Uzbekistan have chosen Kemerovo Region universities to get higher education necessary to develop mining industry in their native countries. Taking higher education courses there they are to study English via Russian as an intermediate language, both languages being foreign to them. To assist themselves in studying the subject, international students from Central Asia use a "crossover" translation technique by means of machine translation at EFL lessons. The assessment of the technique has been carried out and some advice is given to both international students from Central Asia and teachers of EFL. Practical value of the "crossover" translation technique promotes training efficient specialists for mining industry capable to learn and apply advanced technologies for innovative development of Central Asia mining regions.
\end{abstract}

\section{Introduction}

Currently Kemerovo Region universities are steadily strengthening their position in the international education market, offering a wide choice of higher education courses in perspective and relevant specialties in mining industry, ecology, and environmental management for international students from Central Asia mining regions in Tajikistan, Kyrgyzstan, and Uzbekistan. The international students from Central Asia are not Russians by their ethnicity - they are Tajiks, Kyrgyz, Uzbeks, Turkmens, Tatars. Taking Bachelor's, Specialist's, and Master's courses provided by Kemerovo State University (KemSU), the leading university of the region, international students need to study a subject "English as a Foreign Language (EFL)". The purpose of the higher education course of EFL is to train students for its direct practical use in their future professional activity in oral and written business communication as well as interpersonal and cross-cultural contacts. The lack of English language skills complicates the professional communication at the international

${ }^{*}$ Corresponding author: r.islamov87@gmail.com 
level, professional mobility, and possibility to learn and represent new achievements in science and technology.

\section{Materials and methods}

It should be noted that native languages of international students are cognate neither to each other nor to Russian and English, i.e. the linguistic groups differ in phonetics, lexis, grammar, and syntax. Tajik relates to Persian language and Kyrgyz and Uzbek languages are Turkic languages [1-3]. Moreover, Russian is also a foreign language for international students from Central Asia and some of them have poor proficiency in it. This fact leads to additional difficulties in acquiring English language competence since all international students at Kemerovo State University have to study English via Russian as intermediate language. It happens for a number of reasons.

Firstly, the university teachers of the Department of Foreign Languages of KemSU do not speak the native languages of international students. Secondly, international students as native speakers of the Tajik, Kyrgyz, and Uzbek languages make the minority in students' groups at the EFL lessons in comparison with the students whose native language is Russian. Therefore, the teacher can use either English or Russian languages to explain any linguistic phenomena of English. Thirdly, the state normative documents regulating educational programs for EFL contain a requirement to shape a students' skill in "translation and interpretation from a foreign language into Russian and from Russian into foreign language with the respect for standards of lexical equivalence as well as grammatical, syntactic, and stylistic standards". Fourthly, the analysis of English language textbooks used in teaching English in Russian universities has shown that students have to appeal to the Russian language to be able to do a half of exercises (see Table 1). Undoubtedly, the control and monitoring of their progress by a teacher also means the use of Russian.

Table 1. The ratio of exercises involving Russian and exercises without involving Russian in English language textbooks.

\begin{tabular}{|c|c|}
\hline $\begin{array}{c}\text { Exercises involving Russian } \\
\text { international words. }\end{array}$ & $\begin{array}{c}\text { Exercises without involving Russian } \\
\text { Agree or disagree with the following } \\
\text { statements. }\end{array}$ \\
\hline $\begin{array}{c}\text { Read and translate the text consulting the } \\
\text { vocabulary in case of difficulty (given in } \\
\text { Russian). }\end{array}$ & $\begin{array}{c}\text { Fill in the gaps in the text using the } \\
\text { words given in the box. }\end{array}$ \\
\hline $\begin{array}{c}\text { Give the English equivalents for the } \\
\text { following Russian word combinations. }\end{array}$ & Answer the following questions. \\
\hline $\begin{array}{c}\text { Read the following words and their } \\
\text { derivatives. Translate them into Russian. }\end{array}$ & $\begin{array}{c}\text { Divide the text into parts and entitle } \\
\text { each one. Give a short summary of each } \\
\text { part. }\end{array}$ \\
\hline $\begin{array}{c}\text { Match the synonyms / antonyms from } \\
\text { columns A and B. }\end{array}$ & $\begin{array}{c}\text { Match the words in column A with their } \\
\text { explanations in column B. }\end{array}$ \\
\hline $\begin{array}{c}\text { Translate a passage from Russian into } \\
\text { English. }\end{array}$ & $\begin{array}{c}\text { Read the sentences, insert the proper } \\
\text { prepositions into them. }\end{array}$ \\
\hline Guess the word by its definition. & $\begin{array}{c}\text { Complete the sentences using the } \\
\text { necessary information from the text. }\end{array}$ \\
\hline
\end{tabular}

As a result, for the purpose of understanding the content of the given educational texts, international students translate such texts and tasks into their native languages if the Russian language is not required to be checked by a teacher in their progress in English. It 
happens because a student mastering any foreign language unconsciously tries to make up an L2 system (a person's second language) looking for common linguistic bases, adapting (assimilating) new rules to the already-existing L1 competence (a person's first language) [4]. If the Russian language is required to be checked by a teacher in their progress in English, international students translate texts and tasks from their native languages into Russian. The conclusion is drawn on the results of the inquiry conducted among 95 international first-year students of the Institute of Fundamental Sciences and the Institute of Biology, Ecology and Natural Recourses of KemSU.

The ethnic representation of international students who participated in the inquiry was the following: Tajiks -52 students (55\%), Kyrgyz - 22 students (23\%), Uzbeks -15 students $(16 \%)$, Turkmens -2 students $(2 \%)$, Tatars -4 students $(4 \%)$. According to the inquiry, $86 \%$ or 81 international students translate educational texts given in English into their native languages, $74 \%$ or 70 international students admit that they further translate the texts from their native languages into Russian, or make a "crossover" translation, i.e. foreign-language-to-foreign-language translation. It looks like this English $\rightarrow$ Tajik/Kyrgyz/Uzbek $\rightarrow$ Russian .

Moreover, $100 \%$ of students confirm that they translate texts and tasks via translation software, viz. machine translation - Google Translate, offline and online computer dictionaries - Oxford Dictionaries, contextual dictionaries - Reverso Context. This list of translation software is composed in the order of decreasing popularity of this or that service among international students. These language services are not randomly chosen. They are well known to a wide audience (i), run in the form of mobile applications (ii) (it allows to use them easily merely touching the screen of a smartphone or a tablet) but, first and foremost, they process the native languages of international students (iii).

Thus, the purpose of the research is to assess the effectiveness of a "crossover" translation technique done with above-mentioned translation software for international students from Central Asia at EFL lessons at a Russian mining region university.

\section{Results and discussion}

The EFL regulating document at the Department of Foreign Languages of KemSU comprises three sections in EFL teaching: (i) academic English, (ii) professional English, (iii) business English. Each section implies certain thematic and lexical content.

1. Section "Academic English" acquaints students with the system of higher education in English-speaking countries and aims to create a foreign-language readiness of students for the academic mobility and implementation of educational international contacts on the basis of English language communication.

Since the main purpose of EFL is students' acquisition of professional vocabulary and terminology of a particular professional field that, in its turn, serves the basis for the development of students' English reading, speaking, writing, and listening-comprehension skills, teaching EFL focuses mostly on lexical problems; grammar plays secondary and supportive roles. However, providing students with a glossary of related terms is not enough. It is more reasonable to provide them with a context [5] that can assist students to master professional terminology and vocabulary within the thematic net of a particular professional field.

Hereinafter, the implementation of machine translation with some analysis for discussion is considered. The idea is to use a "crossover" technique, i.e. to translate the educational texts from English into international students' native languages and further to translate the obtained outcome into Russian for further analysis. To estimate the final outcome, the following 2-1-0 scale is used: 2 stands for the best match (the outcome is fully 
equivalent), 1 stands for satisfying match (the outcome is partially equivalent), 0 stands for poor match (the outcome is not equivalent). Some extracts of educational texts are given below as well as a list of vocabulary to be learned by the students. Despite the shortness of examples, it is possible to obtain understanding of how translation software processes such texts as well as to get approximate insight into the international students' comprehension.

For example, the first extract under consideration is taken from the topic "Higher Education":

"Higher education in England is provided by colleges, universities and private colleges. Students normally enter higher education from age 18 onwards, and can study for a wide variety of vocational and academic qualifications, including certificates of higher education, bachelor's degrees and integrated master's degree. The typical first degree offered at English universities is the bachelor's degree with honours, which usually lasts for three years, although more vocational foundation degrees, typically lasting two years are also available in some institutions. Many institutions now offer an integrated master's degree, particularly in STEM subjects (Science, Technology, Engineering and Mathematics), as a first degree, which typically lasts for four years, the first three years running parallel to the bachelor's course. While studying for the first degree, students are known as undergraduates".

The academic vocabulary and terminology are represented by 24 words and word combinations. The analysis of equivalency after a "crossover" translation shows that the Tajik variant comprises 3 mistakes (or inaccuracies), the Kyrgyz variant comprises 0 mistakes (or inaccuracies), and the Uzbek variant comprises 0 mistakes (or inaccuracies). Thus, Kyrgyz and Uzbek receive 2 (100\% accuracy), Tajik receives 1 (87.5\% accuracy). Machine translation meets the desired outcome but applying the dictionaries by the students should not be neglected, especially for Tajik language.

2. Section "Professional English" advances the foreign-language communicative competence on the basis of the subject of a particular professional field (in our case computer science, chemistry and ecology). The extract under consideration is from the topic "Computer programming" (for the students majoring in mathematics and computer sciences):

"Computer programming is the process of designing and building an executable computer program for accomplishing a specific computing task. Programming involves tasks such as: analysis, generating algorithms, profiling algorithms' accuracy and resource consumption, and the implementation of algorithms in a chosen programming language (commonly referred to as coding). The source code of a program is written in one or more languages, which is directly executed by the central processing unit. The purpose of programming is to find a sequence of instructions that will automate the performance of a task on a computer, often for solving a given problem. Tasks accompanying and related to programming include: testing, debugging, source code maintenance, implementation of build systems, and management of derived artifacts, such as the machine code of computer programs".

The professional mathematical vocabulary and terminology are represented by 19 words and word combinations. The analysis of equivalency after a "crossover" translation shows that the Tajik variant contains 5 mistakes (or inaccuracies), the Kyrgyz variant contains 2 mistakes (or inaccuracies), and the Uzbek variant contains 4 mistakes (or inaccuracies). Thus, Kyrgyz receives 1 (89.47\% accuracy), Uzbek receives 1 (78.95\% accuracy) and Tajik receives 1 (73.68\% accuracy). As in the previous case, the outcome after machine translation is tending to face the truthfulness but it is strongly recommended to recheck them via dictionaries. What is more, in some cases, it is prohibited to ignore professional dictionaries since some outcome is far from accuracy due to English polysemy. 
The next extract under consideration is from the topic "Chemical reaction" (for the students majoring in chemistry):

"A chemical reaction is a process that leads to the chemical transformation of one chemical substance to another. Classically, chemical reactions encompass changes that only involve the positions of electrons in the forming and breaking of chemical bonds between atoms, with no change to the nuclei. The general concept of a chemical reaction has been extended to reactions between entities smaller than atoms, including nuclear reactions, radioactive decays, and reactions between elementary particles, as described by quantum field theory. The substance initially involved in a chemical reaction is called reactants or reagents. Chemical reactions are usually characterized by a chemical change, and they yield one or more products, which usually have properties different from the reactants. Chemical reactions are described with chemical equations, which symbolically present the starting materials, end products, and sometimes intermediate products and reaction conditions" [6].

The professional chemical vocabulary and terminology are represented by 21 words and word combinations. The analysis of equivalency after a "crossover" translation shows that the Tajik variant contains 8 mistakes (or inaccuracies), the Kyrgyz variant contains 8 mistakes (or inaccuracies), and the Uzbek variant contains 3 mistakes (or inaccuracies). Thus, Uzbek receives 1 (85.71 \% accuracy), Kyrgyz and Tajik receive 1 (61.9\% accuracy). Despite estimated of 1, Kyrgyz and Tajik variants comprise some unacceptable variants of translation. Therefore, the estimation is tending to 0 or 0.5 at least. It is possible to conclude that chemical texts are rather inappropriate for machine translation and students should be careful in using it. Undoubtedly, machine translation must not be highly welcome in studying process and this particular example again proves this statement. It is recommended to use only dictionaries for this case to obtain correct translation.

The following extract is taken from the topic for the students majoring in nature management and is called "Ecology":

"Noxious industrial gases unknown to nature are able to trap the heat of the sun. So, the planet becomes covered by a layer of the gases which keep the heat from getting out. This dense layer of industrial gases produces the global warming. This is also called "the greenhouse effect". Thus, pollution in the atmosphere does not enable heat of the sun to escape back into space. When the temperature of the atmosphere rises, the climatic conditions of different areas on Earth change. Oil spills, effluent from factories and sewages add to pollution of rivers and seas. The destruction of nature gradually led to the loss of the most essential element of existence - a healthy biological habitat. People suffer from their own activity but stay careless: they continue to demand more comfort and do everything possible to obtain it". [7].

The professional ecological vocabulary and terminology are represented by 23 words and word combinations. The analysis of equivalency after a "crossover" translating shows that the Tajik variant comprises 8 mistakes (or inaccuracies), the Kyrgyz variant comprises 5 mistakes (or inaccuracies), and the Uzbek variant comprises 5 mistakes (or inaccuracies). Thus, Uzbek and Kyrgyz receive 1 (78.26\% accuracy), and Tajik receives 1 (65.22\% accuracy). The Tajik variant demonstrates the lower equivalence variants. However, it is not lower than $50 \%$; nevertheless, it should not be recognized as acceptable. In fact, it can be described as poor in some cases. The use of dictionaries is highly recommended.

Nevertheless, the outcome of machine translation cannot be recognized as unacceptable every time. Today's systems of machine translation are quite operable with the scientific texts and there is no outcome lower than $50 \%$ of equivalency. It happens due to the fact that approximately a half of vocabulary contains $60 \%$ of international words. More than that, the professionally-oriented content of EFL at the Institute of Fundamental Sciences of KemSU assumes the attainment of the English vocabulary in such fields as computer technologies, information systems and databases, and natural sciences having no lexical 
equivalents in the native languages of international students. The reasons for the lack of authentic vocabulary and professional terminology in the native languages of international students have some historical roots, among them are the lack of centuries-old written tradition and scientific discoveries in pre-Soviet time that limited the development of languages of the peoples of Central Asia, i.e. they could not acquire own language resources for providing the developing areas of human knowledge and new technologies using Russian for these purposes instead [2]. Moreover, English-language borrowings in the field of computer technologies and natural sciences are also used in Russian language. Therefore, the scientific internationalism and a great number of borrowed words have predetermined the satisfying level of machine translation outcome, which can be even improved via a combined "package" technique with the use of professional dictionaries in addition.

3. Section "Business English" is aimed at shaping and development of communicative skills, which are not directly connected with the specific field of professional activity but they are important for the realization of professional duties under such conditions when these duties assume the international contacts on the basis of foreign-language communication. For example, the business phone communication, writing business letters (the inquiry letter, the letter of response, the order letter (of a product or any equipment), the advertising letter, the letter of complaint with the identification of claims and ways of their settlement, etc.), applying for a job (writing a CV, an application or a portfolio).

The use of a foreign language as a means of cross-cultural and professional and business communication assumes the knowledge of main cultural features, traditions, standards of behavior and etiquette of native speakers. Acquiring this knowledge is necessary for full and productive dialogue of cultures. As a result, we pay much attention to the etiquettical framework of communication and idioms as expressions of sociocultural features of English native speakers.

The assessment of machine translation efficiency used for etiquettical expressions such as Good morning! / Good afternoon! / Good evening! / Good night! / Hello! How are you? / Nice to meet you! / Happy birthday! / Congratulations! / I am sorry! / Please! / Good bye! / Thank you! / Excuse me! / It doesn't matter / Yours sincerely, etc., shows that it is not necessary to use dictionaries. All variants get 2 for the accuracy of "crossover" translation done by Google Translate [5]. It might be easily explained by the fact that the users around the world have an opportunity to take part in advancing and improving machine translation through manual editing that creates a so-called "human-aided translation base", i.e. the more users edit outcome, the better machine translation develops. If compared with the above-mentioned extracts, it is evident that everyday language is more often edited by users than the scientific one.

As for idiomatic expressions, a decision was made to conduct a lesson devoting to the study of English idioms with finding equivalence in Russian, Tajik, Kyrgyz, and Uzbek languages. The chosen idioms are related to the scientific area. All the idioms were considered via Russian language as lingua franca for some comments, definitions, and explanations of the meaning of English expressions to the international and Russian students with the presentation an equivalent in Russian. The idea of such activity is not only in cultural sharing but experimenting with machine translation as well. The question is "If machine translation satisfactorily processes the lexis in most cases, will it be possible to satisfy the cultural aspect of language, i.e. to translate idioms from-and-into all abovementioned languages?"

International students were asked to translate the idioms under consideration into their native languages for better understanding their meanings by means of machine translation. The students were asked to assess the translation according to the following 2-1-0 scale where 2 stands for best match (the idioms are fully equivalent), 1 stands for satisfactory 
match (the sense is clear but equivalency is wrong), 0 stands for poor match (the outcomes are out of equivalency) (see Table 2).

Table 2. The international students' assessment of efficiency of machine translation used for idiomatic expressions.

\begin{tabular}{|l|c|c|c|c|c|c|c|c|c|}
\hline \multirow{2}{*}{ English Idiom } & \multicolumn{3}{c|}{ Tajik } & \multicolumn{3}{c|}{ Kyrgyz } & \multicolumn{3}{c|}{ Uzbek } \\
\hline & $\mathbf{2}$ & $\mathbf{1}$ & $\mathbf{0}$ & $\mathbf{2}$ & $\mathbf{1}$ & $\mathbf{0}$ & $\mathbf{2}$ & $\mathbf{1}$ & $\mathbf{0}$ \\
\hline $\begin{array}{l}\text { 1. Acid test (a conclusive test to } \\
\text { establish quality) }\end{array}$ & & $\checkmark$ & & & & $\checkmark$ & & & $\checkmark$ \\
\hline $\begin{array}{l}\text { 2. Cutting edge (advanced and } \\
\text { innovative) }\end{array}$ & $\checkmark$ & & & & & $\checkmark$ & $\checkmark$ & & \\
\hline $\begin{array}{l}\text { 3. Light years ahead (out in front } \\
\text { with new development or success) }\end{array}$ & & $\checkmark$ & & & & $\checkmark$ & & & $\checkmark$ \\
\hline $\begin{array}{l}\text { 4. Blind with science (confused with } \\
\text { highly technical language) }\end{array}$ & & & $\checkmark$ & & & $\checkmark$ & & & $\checkmark$ \\
\hline $\begin{array}{l}\text { 5. It is not rocket science (easy to do } \\
\text { or understand) }\end{array}$ & & $\checkmark$ & & & $\checkmark$ & & & $\checkmark$ & \\
\hline 6. Up and running (ready for use) & & & $\checkmark$ & & & $\checkmark$ & & & $\checkmark$ \\
\hline
\end{tabular}

The assessment of machine translation efficiency for idiomatic expressions shows that practically all variants of machine translation of the idioms into the native languages of international students have received 0 or 1 since all of them represent the literal translation. Besides, the students note that some words (or word combinations) selected by a machine for translation do not exist in their languages, sentences are syntactically incorrect, and spelling is erroneous. It leads to the distortion of original sense and causes misunderstanding.

International students experience certain difficulties in overcoming a language barrier and immersion in a foreign culture [8]. Educational process in a situation of multicultural communication should be a dialogue with representatives of different cultures, mutual enrichment of languages, students' and teachers' personal fulfillment in an educational environment of a university. Therefore, at EFL lessons in ethnically diverse groups we work in ethnically mixed pairs and small groups for shaping vocabulary and speaking skills since a foreign language becomes a part of the cognitive sphere of a student only as a result of the involvement in the interactions and the relations in the process of communication [9]. Moreover, the organized co-educational activity of students of ethnically diverse groups promotes the freedom from ethnocultural stereotypes and cross-cultural tolerance [10].

\section{Conclusion}

In conclusion it can be stated that the effectiveness of a "crossover" translation technique at "English as a Foreign Language" lessons for international students from Central Asia at a Russian university has been proven for Academic English, Professional English, and Business English Section texts and vocabulary. Thus, "crossover" translation done with machine translation can be recommended to international students from Central Asia to assist them in everyday communication with fellow students, teachers and so forth as well as to translate some academic and professional texts aimed at shaping their English language competence that, in its turn, promotes training efficient specialists for mining industry capable to learn and apply advanced technologies for innovative development of Central Asia mining regions. However, the technique is not effective with some sociocultural language features as it is clearly seen in the case of idioms. Therefore, it is 
advisable not to overuse machine translation at EFL lessons combining it with a "package" technique including the use of dictionaries of all kinds.

\section{References}

1. H. Boeschoten, The Turkic Languages, 1, 357-378 (1998)

2. B. Comrie, The languages of the Soviet Union, 4, 340 (1983)

3. J. Perry, Tajik Persian Reference Grammar, 2, 521 (2005)

4. D. Domalewska, International Journal of Languages, Literature and Linguistics, 1, 6-9 (2015)

5. E. Marinova, N. Rabkina, M. Ryabova, O. Valko, E3S Web Conf., 41, 04040 (2018)

6. IUPAC, Compendium of Chemical Terminology, 2, 01033 (2006)

7. T. Sergeychik, Professional English in Chemistry (KemSU, Kemerovo, 2014)

8. Z. A. Latipov, Revista ESPACIOS, 38:56, 27 (2017)

9. J. J. Thoms, Foreign Language Annals, 45, 8-27 (2012)

10. D. Beyazkurk and J. E. Kesner, International Education Journal, 6, 547-554 (2005) 